\title{
Diagnosing isolated hepatosplenic tuberculosis in an immunocompetent patient: A case report
}

Om Dawani

Michigan State University, East Lansing, MI, USA

Raja Samir Khan

Aga Khan University

Mujtaba Jamal Syed

Dow University of Health Sciences, Karachi, Pakistan

Abdul Moid Shehzad

Dow University of Health Sciences, Karachi, Pakistan

Ahmed Alratoot

Michigan State University, East Lansing, MI, USA

See next page for additional authors

Follow this and additional works at: https://ecommons.aku.edu/pakistan_fhs_mc_med_pulm_critcare

Part of the Pulmonology Commons

\section{Recommended Citation}

Dawani, O., Khan, R. S., Syed, M. J., Shehzad, A. M., Alratoot, A., Ahmed, M. F. (2018). Diagnosing isolated hepatosplenic tuberculosis in an immunocompetent patient: A case report. Tropical Doctor, 48(3), 232-234.

Available at: https://ecommons.aku.edu/pakistan_fhs_mc_med_pulm_critcare/142 
Authors

Om Dawani, Raja Samir Khan, Mujtaba Jamal Syed, Abdul Moid Shehzad, Ahmed Alratoot, and Muhammad Faizan Ahmed

This report is available at eCommons@AKU: https://ecommons.aku.edu/pakistan_fhs_mc_med_pulm_critcare/142 


\title{
Diagnosing Isolated Hepatosplenic Tuberculosis in an Immunocompetent Patient: A Case Report
}

\begin{abstract}
$\underline{\text { Abstract }}$
For many years, Tuberculosis (TB) has been endemic in Pakistan; many rare and unusual presentations have been reported. There are a myriad of non-specific symptoms which always requires a high index of clinical suspicion for TB. WHO data suggests that Pakistan ranks as the $5^{\text {th }}$ highest country burdened with $\mathrm{TB}$ and has the $4^{\text {th }}$ highest prevalence of multi-drug resistant TB globally. With an annual incidence of 277 cases per 100,000, the importance of early diagnosis and treatment is self-evident. We present a case where a strong suspicion of isolated hepatosplenicTB in an immunocompetent patient justified a directed approach.
\end{abstract}

Keywords: Tuberculosis; Isolated; Hepatosplenic Tuberculosis; Immunocompetent; Diagnosis

\section{Case Report}

A 32-year old male presented to the Civil Hospital, Karachi, with complaints of low grade fever and unintentional weight loss of $8 \mathrm{~kg}$ over the previous four months. His fever was reported to be intermittent, and was associated with generalized weakness. His appetite had also decreased. He denied a productive cough, chest pain, abdominal pain, night sweats, and was otherwise asymptomatic. His past medical and family history were insignificant. He was not addicted to drugs norto alcohol and had never smoked.

On examination, severe cachexia was noted. There was no anemia, jaundice, palpable lymphadenopathy, clubbing or oedema. On abdominal examination, an enlarged, firm spleen palpable $2 \mathrm{~cm}$ below the costal margin was palpated, the remaining examination being unremarkable. Initial laboratory screening revealed a haemoglobin level of $130 \mathrm{~g} / \mathrm{l}$, a total leucocyte count of $10.3 \times 10^{9} / \mathrm{L}$ with a differential of $70 \%$ polymorphs, $22 \%$ lymphocytes, and $2 \%$ eosinophils and a platelet count of $346 \times 10^{9} / \mathrm{L}$. The C-reactive protein (CRP) level was raised at $75.6 \mathrm{mg} / \mathrm{L}$ but electrolyte levels, liver and renal function tests were all within normal limits. A blood culture showed no growth.

Further tests for malaria, infectious mononucleosis, hepatitis B \& C, brucellosis and HIV were all negative. The chest radiograph was unremarkable. Abdominal ultrasonography revealed hypodense lesions in the liver and an enlarged spleen of $13.7 \mathrm{~cm}$ size. Computed tomography (CT) abdominal scan of the abdomen confirmed multiple lesions $(9-17 \mathrm{~cm}$ in size) throughout the liver, an enlarged spleen without lesions and small subcentimetric mesenteric lymph nodes. The coagulation profile was normal. After full counselling concerning the risks and advantages, an ultrasound-guided fine needle aspiration biopsy of the hepatic lesions revealed the presence of chronic lymphoplasmacytic inflammation with caseous necrosis consistent with the diagnosis of TB (Figure 1). Acid Fast Bacilli (AFB) smear test and PCR assay for Mycobacterium Tuberculosis and Quantiferon-TB test were all however negative. After appropriate counseling regarding the diagnosis and treatment options available, a trial of antituberculous therapy was initiated, resulting in recovery after six months of treatment without adverse reactions. At 15 months' follow-up, he had gained $>4 \mathrm{Kg}$ in weight and ultrasound scan of liver and spleen showed a reversion to normal. He felt generally better and reported no fever or weaken. 

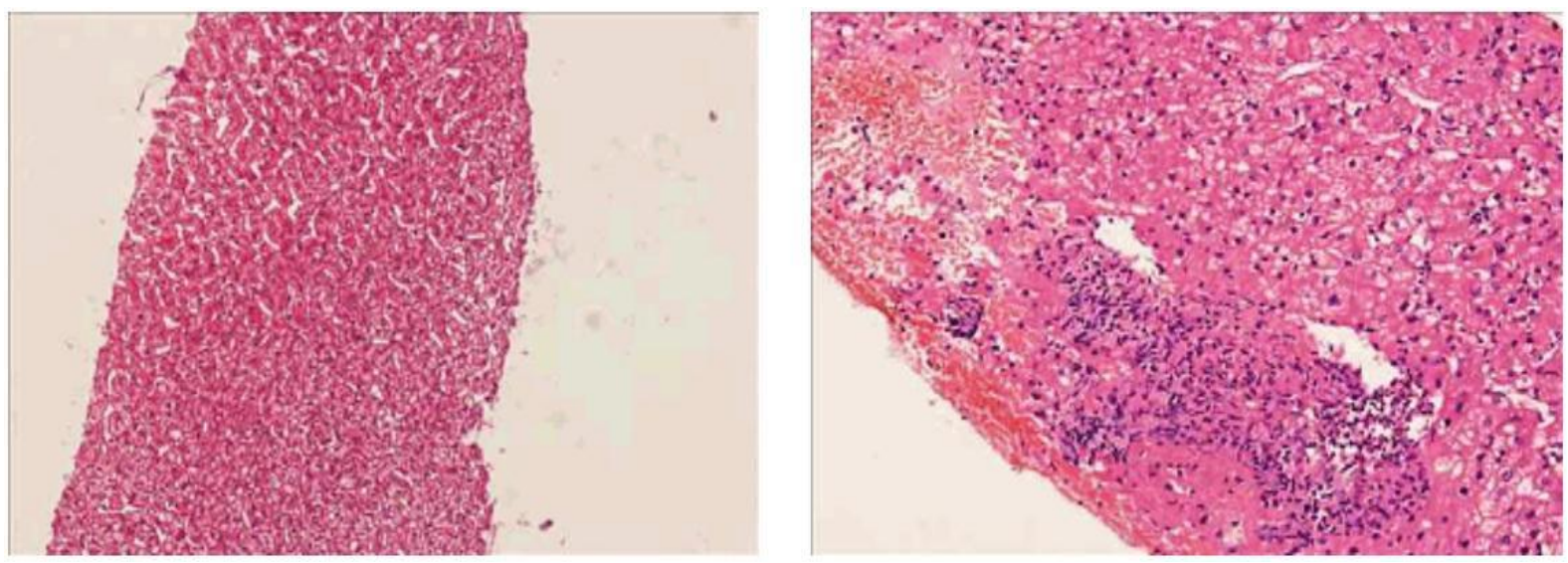

Figure 1: Histological examination of sections from liver biopsy (at 10x magnification) showed degeneration of hepatocytes along with necrotic tissue debris, extensive caseation necrosis and multiple epitheloid granulomas containing Langerhans giant cells. Moderate lymphocytic infiltration was present

\section{Discussion}

Tuberculosis is currently increasing in severity owing to the increasing prevalence of AIDS and the development of multi-drug resistant strains. The rising global burden of this disease has accounted for an increase in unusual case presentations of TB [1].

Our case, initially presenting with non-specific complaints, led initially towards a diagnosis of chronic malaria or primary liver disease. Although TB is of extremely high prevalence in Pakistan, the denial of any cough, productive sputum or night sweats did not suggest TB in the first instance. However, such non-specific symptoms are the chief presenting symptoms described of all hepatosplenic TB patients [2].

The abdominal ultrasound scan confirmed physical findings but did not confirm any specific diagnosis. Thus an abdominal CT scan was ordered but there was a waiting time of two weeks before this could be done.Abdominal exploration was ruled out by the cachectic condition of the patient, and so an ultrasound-guided biopsy was decided upon, as previously described.[2, 3] Whilst a CT-guided biopsy has greater accuracy, and thus less risk of complication, the delay entailed by repeating this scan could not be envisaged. Although a splenic biopsy has a higher diagnostic yield [4], it is riskier than a liver biopsy, which is more frequently performed in our institution.

Despite the absence of acid-fast bacilli, the considered opinion was that TB was nonetheless most likely, as in the similar described [2]. Hepatosplenic TB is a curable disease with relatively good outcomes, but delay in treatment may be fatal. Where a hard and fast diagnosis cannot be reached, it is entirely reasonable to accept the most likely choice.

\section{Conclusion}

Though a trial of TB treatment is frowned upon in some circles, where the diagnosis of TB is correct beyond reasonable doubt, treatment must be started without further delay, especially in a cachectic patient.

\section{References}

1. Purl AS, Nayyar AK, Vij JC. Hepatic Tuberculosis. Ind. J. Tub.1994; 41, 131$134 \underline{\text { PubMed }}$ 
2. Park S, Lee E, Kim T, et al. Primary Hepatosplenic Tuberculosis in an Immunocompetent Adult and Domestic Literature Review. Soonchunhyang Medical Science. 2012;18(2):134137

3. Hasan, R., Kumar, S., Mathew, M. and Kadavigere, R. Isolated hepatosplenic tuberculosis: a rare presentation. BMJ Case Reports 2015; p.bcr2015211065.

4. Kumar PV, Monabati A, Raseki AR, et al. Splenic lesions: FNA findings in 48 cases. Cytopathology 2007;18: 151-6 .

5. Steele K, Schweitzer MA, Sue JL, Kantsevoy SV. Flexible transgastricperitoneoscopy andliver biopsy: a feasibility study in human beings (with video). GastrointestEndosc. 2008; 68 (1): 61-66 